\title{
Density and Mobility Impact on MANET Routing Protocols in a Maritime Environment
}

\author{
Rabab J. Mohsin \\ School of Computer Science and Electronic Engineering \\ University of Essex \\ Colchester, Essex CO4 3SQ, UK \\ AL-Mustansiriya University,College of Engineering \\ Computer and Software Engineering Dept.,Baghdad, Iraq \\ Email: rjmohs@essex.ac.uk
}

\author{
John Woods , Mohammed Q. Shawkat \\ School of Computer Science and Electronic Engineering \\ University of Essex \\ Colchester, Essex CO4 3SQ, UK \\ Email: woodjt@essex.ac.uk,mshawk@essex.ac.uk
}

\begin{abstract}
Mobile Ad-Hoc Networks (MANETS) are multi hop wireless networks, where a packet hops through a number of intermediate nodes within coverage range of each other to reach the intended destination. The novel application of MANET routing protocols in the marine environment using available technology is one of the contributions of this work. The high cost of other available technologies which require direct connection to IP networks make our approach an attractive proposition for small craft. In this paper we investigate the effect of different maritime traffic patterns on the performance of three different MANET routing protocols which are Ad hoc On-Demand Distance Vector (AODV), Ad hoc On-Demand Multi Path Distance Vector (AOMDV) and Destination-Sequenced Distance Vector (DSDV). The traffic patterns are represented by different node densities and mobility behaviours which are likely to be found in the marine environment. Performance evaluation of the MANET protocols is compared in terms of packet delivery ratio.
\end{abstract}

Keywords-Mobile Ad-hoc Networks, Destination-Sequenced Distance Vector, Ad hoc On-Demand Distance Vector, Ad hoc OnDemand Multi Path Distance Vector.

\section{INTRODUCTION}

MANETs are self-organizing networks of mobile nodes without any infrastructure or centralized administration like base stations. Node communication is accomplished via other nodes which are called intermediate or forwarding nodes. Therefore there is a need for a routing procedure between nodes and the routing protocol plays a major role in any MANET [1]. The movement patterns of MANET nodes are characterized by mobility models and each routing protocol shows specic characteristics for these models [2]. In order to decide the most adaptive and efficient routing protocol for the dynamic topologies of MANETs, the routing protocols behaviour needs to be analysed at different node speeds, network size, in addition to node density [3]. The node mobility patterns vary for different scenarios; military adhoc networks exhibit both random and group movement of soldier nodes at low speed, and vehicle nodes (e.g. tanks, trucks etc.) at relatively high speeds. Similarly, the behaviour and movement patterns of nodes in airborne ad-hoc networks are different from military ad-hoc networks [4]. Ship ad-hoc networks on the other hand also have distinctive density and mobility characteristics constrained by various ship density patterns and movement trajectories. In this paper we use the
VHF physical layer previously proposed in [5] to simulate the marine communication environment in the NS2 simulator [6]. The proposed novel VHF physical layer enables marine Ad Hoc multi hop communication using the VHF band already available on most ships eliminating the need for high cost IP networks currently provided by satellite communication. We use this network to investigate the performance of MANET routing protocols under different densities and mobility characteristics particular to the marine environment. We will discuss the advantages and disadvantages of each MANET protocol and compare their performance for different densities and mobility constraints. The rest of this paper is structured as follows. In Section two, we discuss the related work. In section three, we briefly describe an overview of the routing protocols in MANETs. In section four, we discuss the effects of node density and mobility. Then in section five we explain the simulation setup. In section six, we analyse and compare the simulation results. Finally conclusions are presented in section seven.

\section{RELATED WORK}

Much research has been focussed on MANET routing protocol performance under different operational constraints such as energy consumption, node density, mobility patterns, traffic type, network size, quality of service etc. Focusing on density and mobility constraints, in [7] the authors have calculated the hop progress by taking into account the role of node density and obtained the required hop count for a multi-hop path. Based on the result, they discuss the scaling relationship between node density, throughput and delay in multi-hop wireless networks. In [8] the authors provide a framework for specifying the connectivity that reflects the underlying network architecture and protocols. Based on this framework, they define and analyse connectivity requirements for two network architectures which are GAF (Geographic Adaptive Fidelity) with Manhattan routing, mainly proposed for ad-hoc networks and AIMRP (Address-light Integrated MAC and Routing Protocol) which uses tier based routing in sensor networks. Also in [9] the authors disclose an analytical framework for the hop count distribution in a multi hop wireless network with an arbitrary node density. They analyse the average progress per hop and obtain the path connectivity probability in a network. On the other hand in [10] the authors 
present a detailed analytical study of the clustering overhead associated with node mobility in wireless ad hoc networks, and propose a way to understand how changing the node mobility influences the clustering overhead. While in [11] the authors discusses the effect of mobility on parameters such as bit error rate (BER) of a multi-hop route joining a source-destination pair, and minimum required node density of an ad hoc wireless network for complete connectivity.And also reveal their importance in real-life scenarios for average pedestrian and vehicular speeds The Automatic Identification System (AIS) [12] is a data system that transfers packets over a VHF data link (VDL) and enables vessels and shore-based stations to send and receive identification information that can be processed by a computer. This information can assist in location awareness and collision avoidance. The existing system used by ships and vessels in marine environment can upload data to live web browsers via satellite based IP networks but are costly to operate for small vessels.

\section{Routing Protocol Overview}

Routing protocols in MANETS can be classified mainly into two types according to the way routes are maintained in the network [13].

\section{A. Reactive Routing Protocols (On-Demand)}

In reactive or on demand protocols, nodes initiate route discovery throughout the network, only when they want to send packets to the destination. For this purpose, a node initiates a route discovery process through the network. The route discovery process is completed once a route is established or all possible variations have been examined. Once a route has been established, it is maintained by a route maintenance process until either the destination becomes inaccessible along every path from the source or until the route is no longer desired through the use of timers [13] . AODV and AOMDV reactive routing protocols will be used in our simulation.

\section{B. Proactive Routing Protocols (Table-Driven)}

Proactive or Table-Driven protocols maintain routing information even before this information is required. Each node maintains routing information to every other node in the network. Route information is generally stored in routing tables and is periodically updated with any change in the network topology. The protocols that fall under this category maintain different numbers of tables. Also, they are not suitable for large scale networks, because they need to maintain entries for each node in the routing table [14]. The DSDV proactive routing protocol will be used in our simulation.

\section{EFFECT OF Node DENSITY AND MOBILITY IN MANETS}

Apart from the choice of routing protocol, the number of nodes present in the network (node density) and their level of movement (node mobility) also affect the performance of the network. In a sparse network, route establishment may be difficult because the nodes may not be in each others communication range. A dense network may cause increased interference among the nodes in the network. It is also important to study the effect of node mobility on the overall network capacity because high mobility levels may cause frequent link breakages leading to packet drops and delays in establishment of new routes [15].

\section{A. Node Density}

Node density in MANETs mainly effects network connectivity and the routing process in the network as follows.

1) Network Connectivity: Network Connectivity has a big effect on the performance of routing protocols and the quality of network connectivity is fundamental for improving the throughput of the network. The important feature that affects network connectivity and the performance is node density, especially in the high-density cases. The notion of network density has been used in many other works. The network node density is known as the number of network nodes in a unit area. Taking into account the transmission range of every node, let the density of a node in a given region be:

$$
p=N r *(s t / s r)
$$

Where $\mathrm{p}$ represents the node density in the specified areas, $\mathrm{Nr}$ represents the number of nodes in the transmission range of each node, st represents the target area where we want to increase the node density, sr represents the area where the node can transmit information. The transmission range $r$ of each node is usually different in different situations, and may be asymmetric in different directions due to environmental factors [16].

2) Route Process: Node density is also an important factor in the process of route selection and route repair. Increasing node density may bring more opportunities for selecting a route, repairing a route along with other benefits. On the other hand,if nodes are too crowded in the network, the hops between them increase the network overhead, and potentially cause the problem of load imbalance. Therefore using a suitable node density within a realistic situation, network performance can be improved [16] .

\section{B. Node Mobility}

Mobility modelling of nodes in ad hoc networks is an immature science, because there are very few real-world ad hoc networks with which models of node mobility can be compared. When simulating MANETs to measure the performance of various protocols, the majority of studies use the random waypoint mobility model [17]. In this model, nodes are randomly spread in a fixed simulation area. Each node chooses a random destination inside the simulation area and a random speed which it obtains from a given range. Each node moves towards its chosen destination and pauses on arrival for a random period of time within a specified range before selecting a new destination. While this mobility model causes the network topology to change over time, it is often criticized as being unrealistic because actual network nodes in real life networks are not likely to move about in such a random way [18].Also because of the non-uniform node speed distribution, Random Way Point exhibits speed decay [19] . Another issue is that the distribution of nodes is not even in the network field using this model, this is due to bias towards the centre of the simulation area. The bias towards the centre is as a result of the next destination selection by the moving 
nodes. Whereas a node that moves from one point to another usually has to move through the centre [19] .

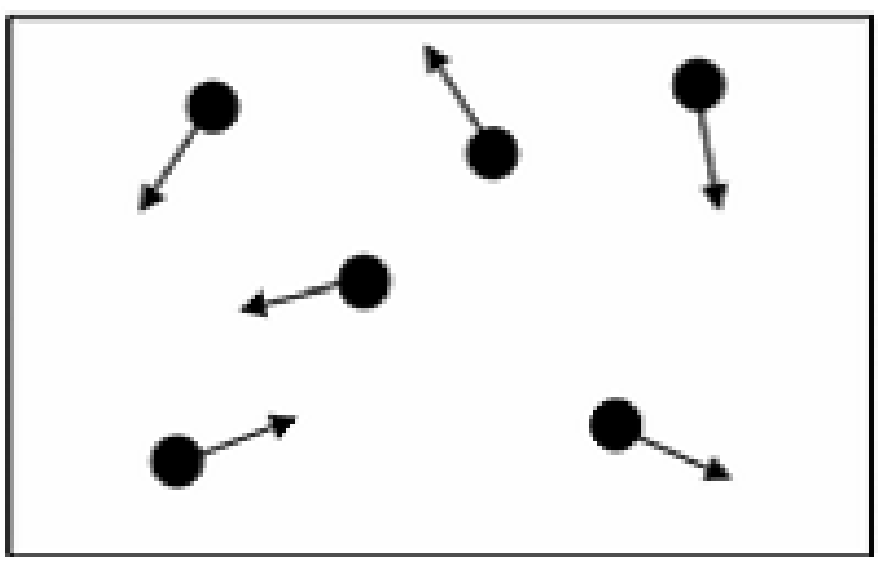

Fig. 1: Random Waypoint Mobility Model [17]

\section{Simulation Setup}

The NS2 simulator version 2.35 [6] has been used to simulate different density and mobility patterns applied to the maritime environment to study the behaviour of three different MANET routing protocols. Also the novel marine physical layer proposed in [5] has been used to simulate marine vessel VHF communication to provide a small vessel Ad-Hoc Network at sea. The traffic source type used in the simulation is CBR (Constant Bit Rate) traffic.The simulation was performed using three MANET routing protocols which are AODV, AOMDV and DSDV. Two types of node transmission ranges have been used: one of $30 \mathrm{Km}$ and another with $40 \mathrm{Km}$. The transmission ranges were calculated using the Free Space Propagation model as shown in equation (2).

$$
p r=\frac{p t * G t * G r *(\lambda)^{2}}{(4 \pi)^{2} * d^{2} * L}
$$

where $\mathrm{Pt}$ is the transmitted power, $\operatorname{Pr}(\mathrm{d})$ is the received power, $\mathrm{Gt}$ is the transmitter antenna gain, $\mathrm{Gr}$ is the receiver antenna gain,d is the Tx-Rx separation and L is the system loss factor [20].

During simulation it was found that NS2 [6] has reliability issues when simulating large areas and therefore the whole simulation is scaled down by a factor of 1000 . This includes the simulation area and all node transmission ranges. We have performed three different simulation scenarios, each with specific density and mobility characteristics as follows:

\section{A. First Scenario}

A random way point mobility pattern was used with different node density values of 50,100,150 and 200 nodes respectively.The simulated nodes move within an area of 400 x $300 \mathrm{Km}$ during a period of 80 Seconds simulation time, during which the nodes pause every 10 seconds to change direction.The data bandwidth for node communication used is $9.6 \mathrm{~Kb} / \mathrm{Sec}$. Table I shows a summary of the simulation parameters used in this simulation.
TABLE I: Simulation Parameters for Scenario 1

\begin{tabular}{|l|l|}
\hline Indicator & Value \\
\hline Simulator & NS2.35 \\
\hline Routing Protocols & AODV, DSDV,AOMDV \\
\hline Simulation Time & $80 \mathrm{sec}$ \\
\hline Traffic Type & CBR \\
\hline Pause Time & $10 \mathrm{Sec}$ \\
\hline No of Nodes & $50,100,150,200$ \\
\hline Simulation Area & $400 \times 300 \mathrm{Km}$ \\
\hline Propogation Model & Free Space \\
\hline Transmission Range & $30 \mathrm{Km}, 40 \mathrm{Km}$ \\
\hline Node Movement Model & Random Way Point \\
\hline Bandwidth & $9.6 \mathrm{~Kb}$ \\
\hline Data Payload & Bits/Sec \\
\hline
\end{tabular}

\section{B. Second Scenario}

In this scenario we have used real ship locations by simulating 60 static nodes located in the English Channel between Clacton on sea in the UK and Middelburg in France over a simulation area of $200 \times 200 \mathrm{Km}$ as in Figure 2,the ship locations were extracted from the real live AIS data website in [21]. TableII shows a summary of the simulation parameters used in this simulation.

TABLE II: Simulation Parameters for Scenario 2

\begin{tabular}{|l|l|}
\hline Indicator & Value \\
\hline Simulator & NS2.35 \\
\hline Routing Protocols & AODV, DSDV,AOMDV \\
\hline Simulation Time & $80 \mathrm{sec}$ \\
\hline Traffic Type & CBR \\
\hline No of Nodes & 60 nodes with real sea locations \\
\hline Simulation Area & $200 \times 200 \mathrm{Km}$ \\
\hline Propogation Model & Free Space \\
\hline Transmission Range & $30 \mathrm{Km}, 40 \mathrm{Km}$ \\
\hline Node Movement Model & Static (no mobility) \\
\hline Bandwidth & $9.6 \mathrm{~Kb}$ \\
\hline Data Payload & Bits/Sec \\
\hline
\end{tabular}

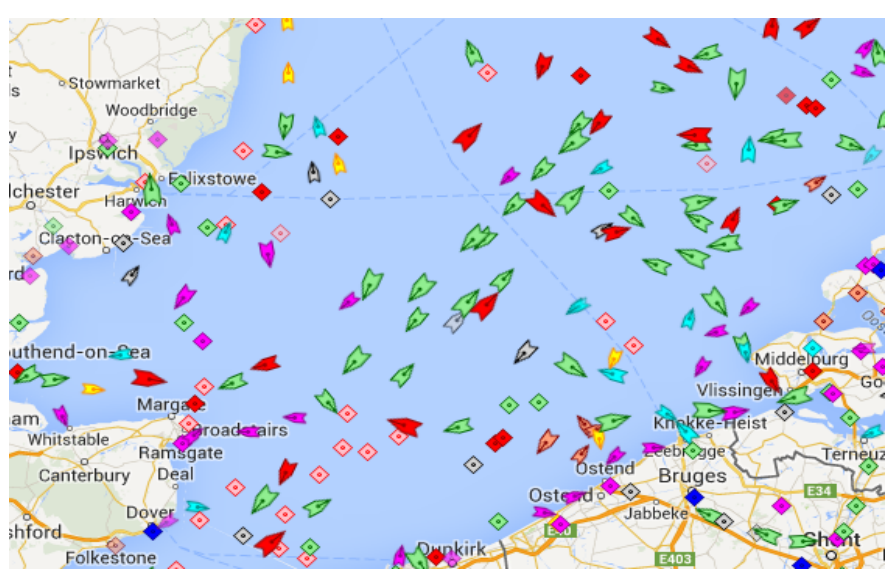

Fig. 2: Real Ship Locations between Clacton and Middleburg (on 1 July at 2:00PM) 


\section{Third scenario}

In this scenario we have used the same parameters as scenario 2 with dense, real mobility patterns extracted from the AIS data website in [21].The mobility patterns extracted consist of real ship speed and direction. Table III shows a summary of the simulation parameters used.

TABLE III: Simulation Parameters for Scenario 3

\begin{tabular}{|l|l|}
\hline Indicator & Value \\
\hline Simulator & NS2.35 \\
\hline Routing Protocols & AODV, DSDV,AOMDV \\
\hline Simulation Time & $80 \mathrm{sec}$ \\
\hline Traffic Type & CBR \\
\hline No of Nodes & 60 nodes with real sea locations \\
\hline Simulation Area & $200 \mathrm{X} 200 \mathrm{Km}$ \\
\hline Propogation Model & Free Space \\
\hline Transmission Range & $30 \mathrm{Km}, 40 \mathrm{Km}$ \\
\hline Node Movement Model & Real mobility patterns extracted from live AIS data \\
\hline Bandwidth & $9.6 \mathrm{~Kb}$ \\
\hline Data Payload & Bits/Sec \\
\hline
\end{tabular}

\section{Fourth Scenario}

In this scenario we have simulated the vessel traffic in the North Sea Figure 3 which represents a sparser network environment than the previous scenarios. 30 mobile nodes were simulated with real vessel locations, speeds and directions also extracted from the real AIS data website in [21] as in scenarios 2 and 3.An overall simulation area of $300 \times 300 \mathrm{Km}$ was used. TableIV shows a summary of the simulation parameters used.

TABLE IV: Simulation Parameters for Scenario 4

\begin{tabular}{|l|l|}
\hline Indicator & Value \\
\hline Simulator & NS2.35 \\
\hline Routing Protocols & AODV, DSDV,AOMDV \\
\hline Simulation Time & $80 \mathrm{sec}$ \\
\hline Traffic Type & CBR \\
\hline No of Nodes & 30 nodes with real sea locations \\
\hline Simulation Area & $300 \mathrm{X} 300 \mathrm{Km}$ \\
\hline Propogation Model & Free Space \\
\hline Transmission Range & $30 \mathrm{Km}, 40 \mathrm{Km}$ \\
\hline Node Movement Model & $\begin{array}{l}\text { Real mobility patterns extracted } \\
\text { from live AIS data }\end{array}$ \\
\hline Bandwidth & $9.6 \mathrm{~Kb}$ \\
\hline Data Payload & Bits/Sec \\
\hline
\end{tabular}

\section{RESUlTS AND COMPARISON}

Performance evaluation of the MANET routing protocols are compared in terms of Packet Delivery Ratio PDR, where PDR is the ratio of data packets that arrive successfully at the destination.Figure4 shows the packet delivery ratio for the three protocols vs. the number of nodes in the first simulation scenario.

It can be seen that there is a clear increase in PDR as the node density increases in the network. The AOMDV protocol records the highest PDR rates and is mostly affected by increasing node density moving from $22 \%$ PDR when simulating

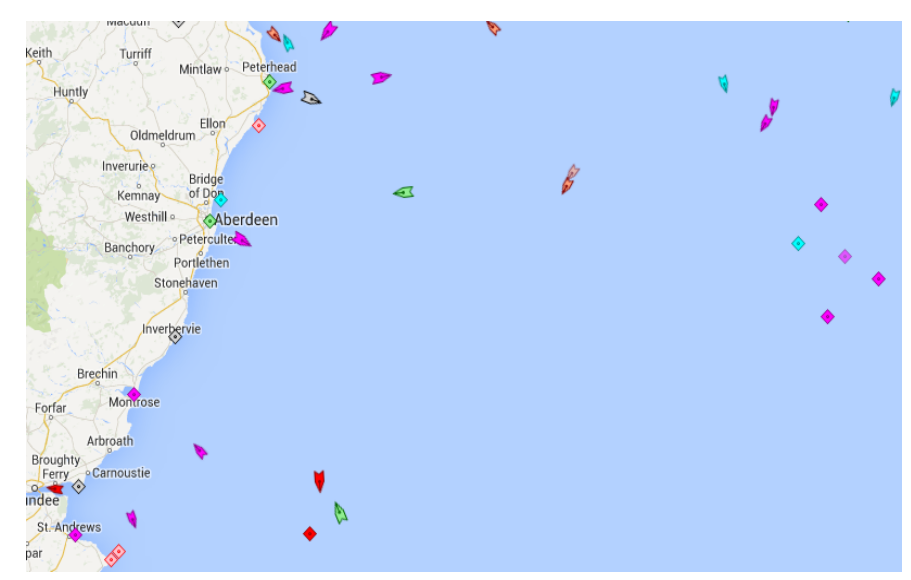

Fig. 3: Real Ship Locations in the North Sea(on 3th of July at 11:00AM)

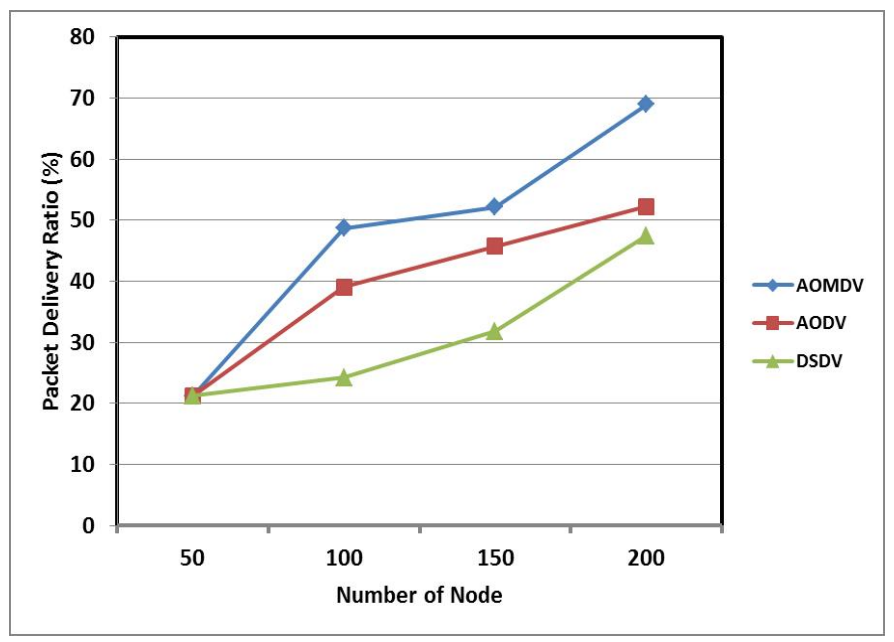

Fig. 4: Packet delivery ratio in Scenario 1

50 nodes to $68 \%$ PDR when simulating 200 nodes.AOMDV was followed by AODV and then DSDV with the lowest rates. Recall that the results in this figure were found using random waypoint mobility. On the other hand figure5 shows the packet delivery ratio for the three protocols vs time in the second simulation scenario.

From the figure,when using real AIS data [12] for determining node density in the absence of mobility, high PDR rates are seen. The AOMDV protocol shows PDR results approaching $100 \%$. This is because the navigational routes drawn by most vessels at sea follow pathways convenient for packet hopping towards the destination. While for the third simulation scenario,PDR rates decline slightly when real mobility patterns are applied to the nodes in scenario 2 reaching a minimum PDR of $80 \%$ with the DSDV protocol.Figure6 shows the PDR vs. time in the third simulation scenario.

Figure 7 shows the packet delivery ratio for the three protocols vs. time in the fourth and last simulation scenarios.The figure clearly shows the effect of sparseness in the network, where as sparseness in the network increases, PDR decreases accordingly because there is an increased chance 


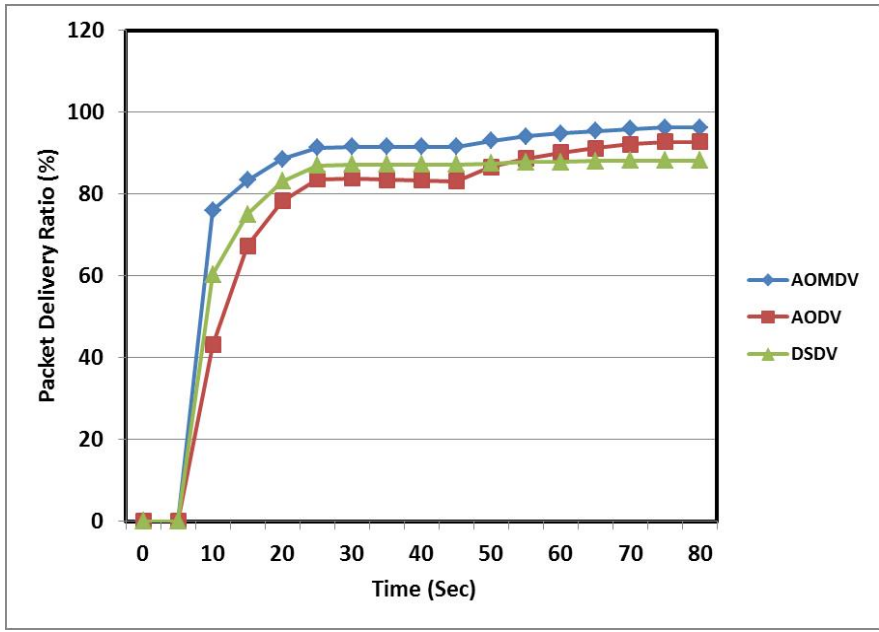

Fig. 5: Packet delivery ratio in Scenario 2

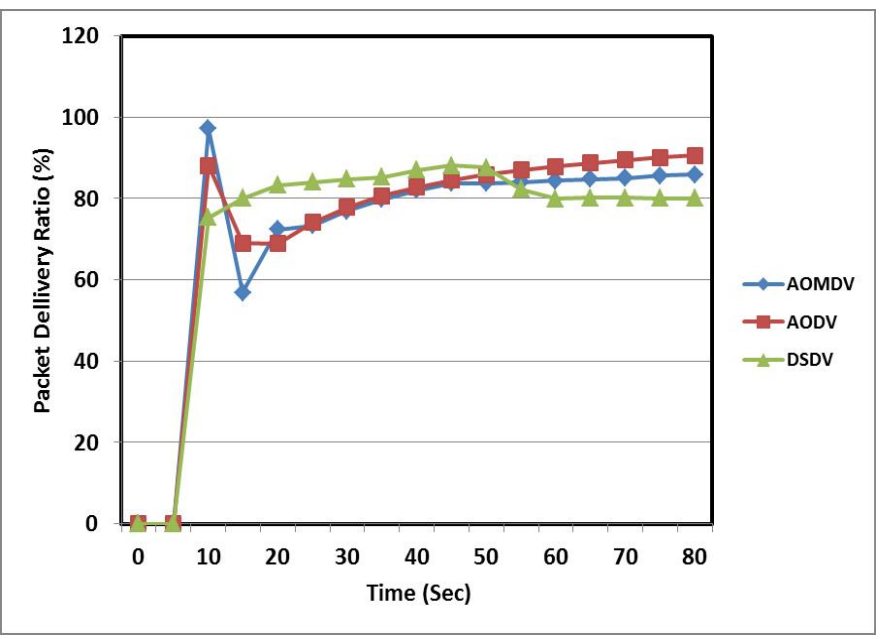

Fig. 6: Packet delivery ratio in Scenario 3

a node will be out of the transmission range. PDR for the AOMDV protocol records the highest rate reaching just under $75 \%$ while DSDV records the lowest rate reaching approximately 58\%. Considering the VHF marine communication environment proposed and the performance of the routing protocols applied in this paper, it can be seen that the performance of MANETs in the marine environment decreases with low density situations where huge portions of sea have few vessels that can be used as multi hops to forward data packets. The optimum solution would be to make each ship retain packets for as long as possible until another suitable candidate is available to pass the packet onto. This leads us to the concept of delay tolerant networking [22] where a routing protocol takes into consideration such sparse networks and works with large buffer sizes and extended packet life times. It can be seen from our simulation results that the marine environment is not always sparse. There are certain areas that have a large density of shiping especially near the shore and on major shipping routes. DTN routing protocols do not perform in dense locations as well as they do in sparse ones [23], therefore DTN routing protocols may not always be suitable.
As a solution, a hybrid routing protocol may be used that has the ability to differentiate between dense and sparse locations and act accordingly. The protocol would initiate the AOMDV functionality in dense locations and DTN functionality in sparse locations, maintaining high adaptability in a diverse environment. In addition to the aforementioned, dealing with data packets may be difficult due to their relatively small size, especially when connectivity opportunities are very limited in a sparse network and maximum data utilisation is desired. Therefore the use of data bundles may be introduced [24].

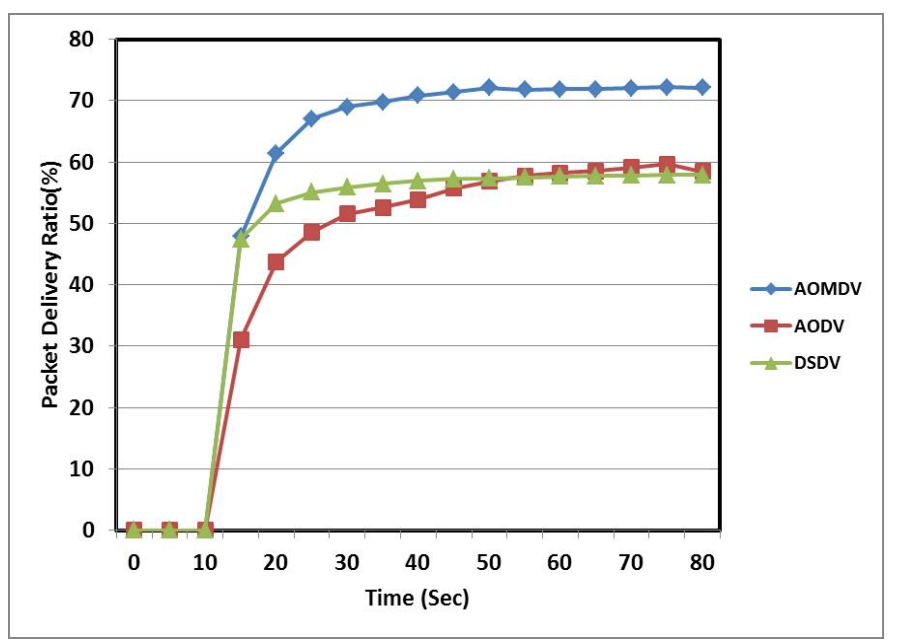

Fig. 7: Packet delivery ratio in Scenario 4

\section{CONCLUSIONS}

We propose the novel application of MANETs in the marine environment and explore the use of routing protocols to achieve a low cost AIS type system using existing on-board equipment. Our solution is aimed at low cost small vessels. The impact of marine traffic patterns on the performance of MANET routing protocols has been investigated.The results show that real-life marine density and mobility patterns show better PDR rates than the random waypoint mobility model. This is because the navigational routes drawn by most vessels at sea tend to follow pathways which aid multi hop connectivity for data packets hopping towards the destination. This is not always the case as there are ships can be scattered sparsely so such pathways do not exist.It can also be concluded that in high density and mobility environments, AOMDV is a better choice than AODV and DSDV for marine applications. Finally it can be concluded that MANET protocol performance has a positive relationship with density and an inverse relationship with mobility and sparseness. PDR rates increase when density increases and on the contrary decrease when mobility or sparseness increases.

\section{REFERENCES}

[1] S. Preethi and B. Ramachandran, "Energy efficient routing protocols for mobile adhoc networks," in Emerging Trends in Networks and Computer Communications (ETNCC), 2011 International Conference on. IEEE, 2011, pp. 136-141.

[2] T. Camp, J. Boleng, and V. Davies, "A survey of mobility models for ad hoc network research," Wireless communications and mobile computing, vol. 2, no. 5, pp. 483-502, 2002. 
[3] F. Maan and N. Mazhar, "Manet routing protocols vs mobility models: A performance evaluation," in Ubiquitous and Future Networks (ICUFN), 2011 Third International Conference on. IEEE, 2011, pp. 179-184.

[4] G. Lin, G. Noubir, and R. Rajaraman, "Mobility models for ad hoc network simulation," in INFOCOM 2004. Twenty-third AnnualJoint Conference of the IEEE Computer and Communications Societies, vol. 1. IEEE, 2004.

[5] R. Mohsin and J. Woods, "Performance evaluation of MANET routing protocols in a maritime environment," in Sixth Computer Science and Electronic Engineering Conference 2014 (CEEC'14). Colchester, Essex, United Kingdom: IEEE, Sep. 2014.

[6] "The Network Simulator - NS-2." [Online]. Available: http://www.isi.edu/nsnam/ns/

[7] J.-C. Kuo, W. Liao, and T.-C. Hou, "Impact of node density on throughput and delay scaling in multi-hop wireless networks," Wireless Communications, IEEE Transactions on, vol. 8, no. 10, pp. 5103-5111, 2009.

[8] S. S. Kulkarni, A. Iyer, C. Rosenberg, and D. Kofman, "Routing dependent node density requirements for connectivity in multi-hop wireless networks," in Global Telecommunications Conference, 2004 GLOBECOM'04. IEEE, vol. 5. IEEE, 2004, pp. 2890-2896.

[9] J.-C. Kuo and W. Liao, "Hop count distribution of multihop paths in wireless networks with arbitrary node density: Modeling and its applications," Vehicular Technology, IEEE Transactions on, vol. 56, no. 4, pp. 2321-2331, 2007.

[10] Y. Gu, R. V. Prasad, and I. Niemegeers, "Analyzing the effect of node mobility in clustered wireless ad hoc networks," in Global Telecommunications Conference, 2009. GLOBECOM 2009. IEEE. IEEE, 2009, pp. 1-6.

[11] M. Bhatt, R. Chokshi, S. Desai, S. Panichpapiboon, N. Wisitpongphan, and O. K. Tonguz, "Impact of mobility on the performance of ad hoc wireless networks," in Vehicular Technology Conference, 2003. VTC 2003-Fall. 2003 IEEE 58th, vol. 5. IEEE, 2003, pp. 3025-3029.

[12] K. Schwehr and P. McGillivary, "Marine ship automatic identification system (ais) for enhanced coastal security capabilities: An oil spill tracking application," in OCEANS 2007. IEEE, Sept 2007, pp. 1-9.

[13] A. K. Gupta, H. Sadawarti, and A. K. Verma, "Performance analysis of aodv, dsr \& tora routing protocols," IACSIT international journal of Engineering and Technology, vol. 2, no. 2, pp. 226-231, 2010

[14] S. Mohseni, R. Hassan, A. Patel, and R. Razali, "Comparative review study of reactive and proactive routing protocols in manets," in Digital Ecosystems and Technologies (DEST), 2010 4th IEEE International Conference on. IEEE, 2010, pp. 304-309.

[15] H. Thriveni, G. M. Kumar, and R. Sharma, "Performance evaluation of routing protocols in mobile ad-hoc networks with varying node density and node mobility," in Communication Systems and Network Technologies (CSNT), 2013 International Conference on. IEEE, 2013, pp. 252-256.

[16] J. Zuo, Y. Wang, Y. Liu, and Y. Zhang, "Performance evaluation of routing protocol in vanet with vehicle-node density," in Wireless Communications Networking and Mobile Computing (WiCOM), 2010 6th International Conference on. IEEE, 2010, pp. 1-4.

[17] J. Yoon, M. Liu, and B. Noble, "Random waypoint considered harmful," in INFOCOM 2003. twenty-second annual joint conference of the IEEE computer and communications. IEEE societies, vol. 2. IEEE, 2003, pp. 1312-1321.

[18] D. Raymond, I. Burbey, Y. Zhao, S. Midkiff, and C. P. Koelling, "Impact of mobility models on simulated ad hoc network performance," in Proc. 9th International Symposium on Wireless Personal Multimedia Communications (WPMC), 2006, pp. 398-402.

[19] K. Amjad and A. J. Stocker, "Impact of node density and mobility on the performance of aodv and dsr in manets," in Communication Systems Networks and Digital Signal Processing (CSNDSP), 2010 7th International Symposium on. IEEE, 2010, pp. 61-65.

[20] A. Mitra, "Lecture notes on mobile communication," A Curriculum Development Cell project Under QIP, IIT Guwahati, 2009.

[21] F. Inc., "Live marine traffic." [Online]. Available: https://www.marinetraffic.com/en/
[22] R. Mangrulkar and M. Atique, "Routing protocol for delay tolerant network: A survey and comparison," in Communication Control and Computing Technologies (ICCCCT), 2010 IEEE International Conference on. IEEE, 2010, pp. 210-215.

[23] H.-M. Lin, Y. Ge, A.-C. Pang, and J. S. Pathmasuntharam, "Performance study on delay tolerant networks in maritime communication environments," in OCEANS 2010 IEEE-Sydney. IEEE, 2010, pp. 1-6.

[24] L. Lambrinos, C. Djouvas, and C. Chrysostomou, "Applying delay tolerant networking routing algorithms in maritime communications," in World of Wireless, Mobile and Multimedia Networks (WoWMoM), 2013 IEEE 14th International Symposium and Workshops on a. IEEE, 2013, pp. 1-6. 